\title{
Synthesis of monodisperse crosslinked polystyrene microspheres
}

\author{
Jiang Kai, Chen Sheng-Li *, Dong Peng and Liu Renxiao \\ State Key Laboratory of Heavy Oil Processing, School of Chemical Science \& Engineering, China University of Petroleum, \\ Beijing 102249, China
}

\begin{abstract}
Monodisperse crosslinked polystyrene (CPS) particles were prepared through the normal emulsion polymerization method by adding crosslinker-divinylbenzene (DVB) into the reaction system after polystyrene (PS) particles grew to $\sim 80 \%$ of the final size. When the amount of crosslinker DVB added was less than $6.17 \mathrm{wt} \%$ based on styrene, the prepared CPS particles were spherical and uniform and the size of the CPS particles could be predicted through the normal emulsion method. The glass transition temperature $(T \mathrm{~g})$ of the prepared CPS particles was higher than that of un-crosslinked PS particles and, the more crosslinker that was added, the higher the $T \mathrm{~g}$ of CPS Particles. The prepared CPS particles had strong resistance to organic solvents.
\end{abstract}

Key words: Emulsion polymerization, crosslinked polystyrene particle, monodisperse particles

\section{Introduction}

Crosslinked polystyrene (CPS) particles are widely used in such fields as ion exchange resin, polymer reagent, polymer catalyst, macromolecular adsorbent and polymer support materials, because of their high specific surface area, good mechanical strength, high surface reactivity and uniformity in size (Huang et al, 2003; Zhang et al, 2001; Zhang et al, 2002). When used as particles of ion exchange resins or adsorbents, the open framework of the crosslinked structure provides good accessibility for function groups. The crosslinked structure is also important for micro-size standard materials which require resistance to dissolution in organic solvents. Therefore synthesis of monodisperse crosslinked polystyrene microspheres is an important research area in the macromolecular field (Chen et al, 1990).

Many methods are available for CPS particles synthesis, and the size and size-distribution of CPS particles are different for different methods. Monodisperse CPS particles prepared by the dispersion-polymerization-method have a diameter of 1-10 $\mu \mathrm{m}$ (Zhang et al, 2001) with divinyl benzene (DVB) (Jun et al, 2003) or 3-(trimethoxysilyl)propyl methacrylate (TMSPM) (Choi et al, 2002) used as crosslinker. The amount of crosslinker used in the synthesis of CPS particles by the dispersion method could be up to $20 \mathrm{wt} \%$ (Choi et al, 2002). Larger particles (diameter $>10 \mu \mathrm{m}$ ) could be obtained through the traditional suspension polymerization method with divinyl benzene as cross-linking agent, but the prepared particles are not uniform in size (You et al, 1994; Xu et al, 1994). Fully crosslinked stable polystyrene particles, with a diameter from 3.5 to $2.8 \mu \mathrm{m}$ (Shim et al, 2004), were prepared by the precipitation polymerization

*Corresponding author. email: slchen@cup.edu.cn

Received March 2, 2008 method, with different divinyl benzene contents from 5 to $75 \mathrm{~mol} \%$. Emulsion polymerization is the most common method for synthesis of monodisperse PS particles having a diameter of 100-1000 nm. The emulsion polymerization was carried out in water, using potassium persulfate as initiator and sodium p-styrene sulfonate as emulsifier. The emulsion polymerization method for synthesis of non-crosslinked PS was used here in this work for synthesizing CPS particles. During the synthesis of CPS particles, the crosslinking agentDVB was added to the reaction solution after the PS particles were formed, so that the crosslinking reaction occurred only on the outer layer of the polystyrene particles. Therefore the addition of the crosslinking agent did not change the final particle diameter, and the reaction conditions (well studied for synthesis of non-crosslinked PS particles) could be used directly for the synthesis of CPS and prediction of their size. Another advantage of this modified emulsion polymerization method is that in comparison with the conventional wholeparticle-crosslinking process, a high degree of crosslinking on the surface of PS particles can be achieved when the same amount of crosslinker is used, without any impact on the spherical morphology of the particles.

\section{Experimental}

Uniform PS particles were synthesized by the emulsion method as follows: $150 \mathrm{ml} \mathrm{H} \mathrm{H}_{2} \mathrm{O}, 18.4 \mathrm{ml}$ styrene, 0.0833 g $\mathrm{NaHCO}_{3}$ and a specific amount of $\mathrm{C}_{8} \mathrm{H}_{7} \mathrm{SO}_{3} \mathrm{Na}$ (sodium styrene sulfonate) were added into a magnetically stirred 300 $\mathrm{ml}$ reactor. The reactor was kept at $75 \pm 0.1{ }^{\circ} \mathrm{C}$, and stirred at $\sim 250 \mathrm{rpm}$. Polymerization was initiated by adding $0.083 \mathrm{~g}$ of $\mathrm{K}_{2} \mathrm{~S}_{2} \mathrm{O}_{8}$ into the reactor and under nitrogen. Polymerization was completed in approximately 10 hours. During the reaction, the variation of PS particles diameter with time was determined with dynamic light scattering (DLS) on Malvern 
Zetasizer Nanosizer. The content of PS particles in aqueous solution was approximately $12 \mathrm{wt} \%$. For the preparation of uniform CPS particles, DVB was added to the reaction solution after particle size reached $\sim 80 \%$ of the final size. The topography of the particles was examined with a HITACHI H-800 transmission electron microscope (TEM).

To obtain pure dry CPS powder, the obtained particles suspension was first deionized with ion exchange resin beads, making the ionic strength of the CPS particles suspension less than $16 \mu \mathrm{s} / \mathrm{cm}$, and then the suspension was freeze-dried. Differential scanning calorimetry (DSC) and thermogravimetric (TG) experiments were performed on a STA409PC Luxx thermal analyzer with a temperature rise rate of $10^{\circ} \mathrm{C} / \mathrm{min}$ and $\mathrm{Ar}$ as purging gas.

\section{Results and discussion}

To determine the appropriate time when the crosslinker should be added to the synthesis system, non-crosslinked PS particles were prepared without cross-linker, and the diameter of PS particles was followed by the DLS technique. As shown in Fig. 1, the final diameter of the PS particles prepared without crosslinker was $\sim 145 \mathrm{~nm}$, and it needed approximately $70 \mathrm{~min}$ for the particles to grow up to $\sim 80 \%$ of the final size. Therefore for the preparation of crosslinked PS particles, the crosslinker, DVB, could be added to the reaction system 70 minutes after the reaction had been started by adding the potassium persulfate initiator. Therefore cross linking occurred only on the shell of the CPS particles and the CPS particle was just like an egg consisting of yolk and egg-white, as shown in Fig. 2. So the degree of outer shell crosslinking was higher than that of whole-particles crosslinked PS when the amount of crosslinker was the same.

Table 1 shows the diameter and monodispersity of CPS particles prepared by using different amounts of DVB crosslinker. The diameter and monodispersity of the CPS particles were measured with a Malvern Zetasizer Nanosizer. When the amount of DVB varied from $1 \mathrm{wt} \%$ to $6.17 \mathrm{wt} \%$, the diameter of CPS particles remained unchanged and the obtained particles were uniform, implying a constant number of PS particles during the growth period. When the amount of DVB reached $7.79 \mathrm{wt} \%$, the size of CPS particles began to increase and the particles were not uniform, indicating a decreasing number of PS particles and aggregation of CPS particles. As a consequence, when the amount of DVB was less than $6.17 \mathrm{wt} \%$, the crosslinker DVB did not interrupt the growth process and monodisperse spherical CPS particles could be obtained, and their size could be predicted from the preparation conditions of emulsion polymerization. However, when the amount of DVB was more than $7.79 \mathrm{wt} \%$, the DVB crosslinker induced aggregation of PS particles and the prepared CPS particles were neither spherical, nor uniform. The size and topography of CPS particles were also observed with TEM, as shown in Fig. 3. The TEM further confirmed the above results obtained from DLS. When the amount of DVB reached $\sim 30 \mathrm{wt} \%$, the synthesis system was unstable and flocculent PS materials were obtained.

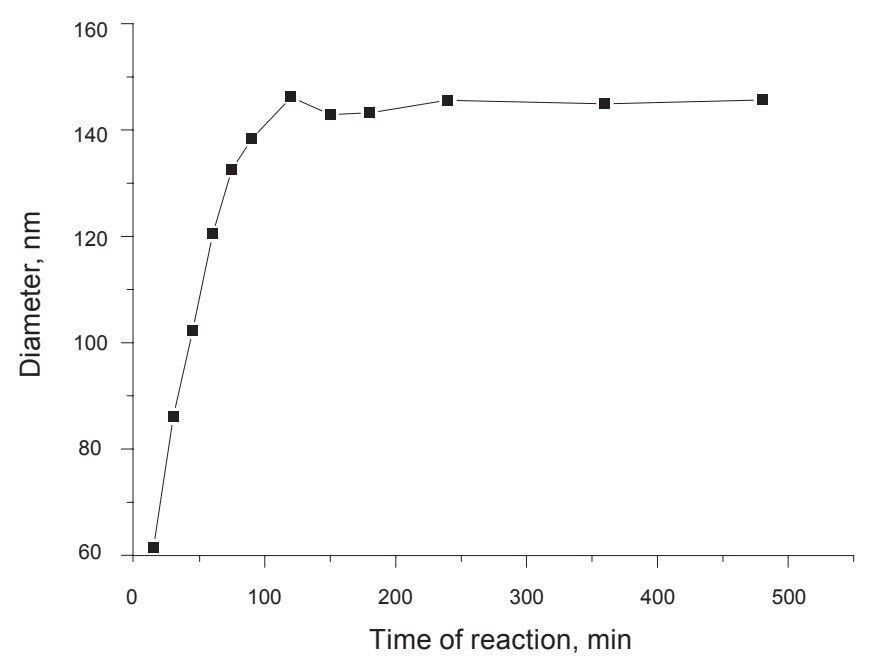

Fig. 1 Variation of PS diameter with time (preparation conditions: $10 \mathrm{wt} \%$ styrene, 0.05 wt $\% \mathrm{NaHCO}_{3}, 0.59 \mathrm{wt} \% \mathrm{C}_{8} \mathrm{H}_{7} \mathrm{SO}_{3} \mathrm{Na}, 0.05 \mathrm{wt} \% \mathrm{~K}_{2} \mathrm{~S}_{2} \mathrm{O}_{8}$ )

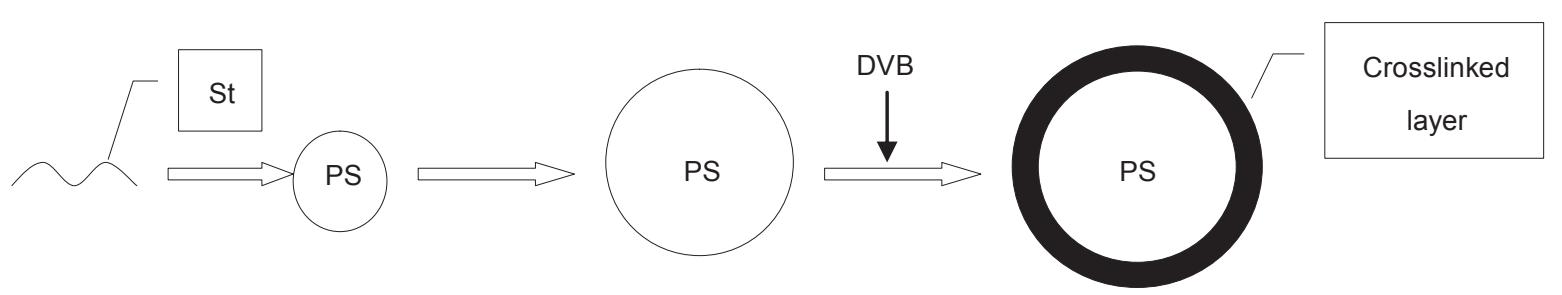

Fig. 2 Schematic diagram of formation of CPS

Table 1 Diameter and polydispersion index of CPS prepared by using different amounts of DVB based on styrene

\begin{tabular}{cccc}
\hline No & DVB, wt $\%$ & Average diameter, nm & Polydispersion index \\
\hline 1 & 1.07 & 195.2 & 0.005 \\
2 & 2.09 & 142.9 & 0.021 \\
3 & 4.24 & 135.7 & 0.008 \\
4 & 6.17 & 175.1 & 0.035 \\
5 & 7.79 & 230.4 & 0.180 \\
\hline
\end{tabular}

Notes: Other preparation conditions: $10 \mathrm{wt} \%$ styrene, $0.05 \mathrm{wt} \% \mathrm{NaHCO}_{3}, 0.59 \mathrm{wt} \% \mathrm{C}_{8} \mathrm{H}_{7} \mathrm{SO}_{3} \mathrm{Na}$ and $0.05 \mathrm{wt} \% \mathrm{~K}_{2} \mathrm{~S}_{2} \mathrm{O}_{8}$ 

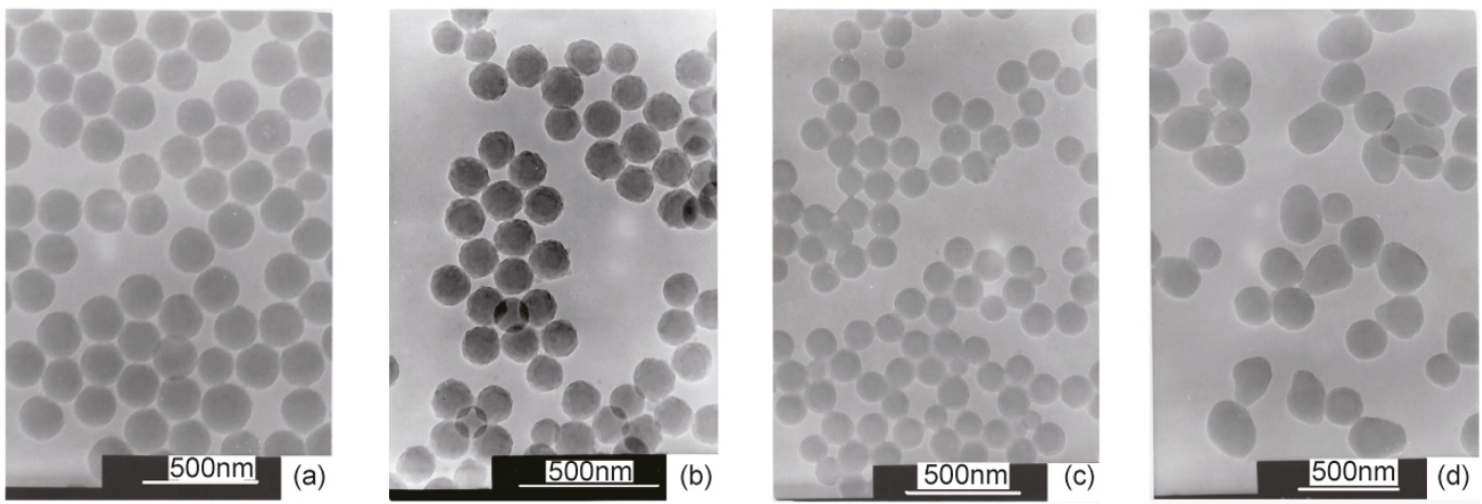

Fig. 3 TEM of CPS prepared with different amounts of crosslinker. a: 1.07 wt $\%$, b: 2.09 wt $\%$, c: 4.24 wt $\%$ and d: 7.79 wt $\%$ (other preparation conditions: $10 \mathrm{wt} \%$ styrene, $0.05 \mathrm{wt} \% \mathrm{NaHCO}_{3}, 0.59 \mathrm{wt} \% \mathrm{C}_{8} \mathrm{H}_{7} \mathrm{SO}_{3} \mathrm{Na}, 0.05 \mathrm{wt} \% \mathrm{~K}_{2} \mathrm{~S}_{2} \mathrm{O}_{8}$ )

The size of PS particles prepared with the emulsion method mainly depended on the concentration of the emulsifier used. Different amounts of sodium p-styrene sulfonate were used in the preparation of CPS and different sizes of CPS particles were obtained. As shown in Table 2, the diameter of CPS decreased with the increase of emulsifier concentration. The more the sodium p-styrene sulfonate, the more the colloidal micelle of styrene, which would form the PS particles through polymerization, so that a larger number of CPS particles were obtained. Therefore, smaller CPS particles could be obtained when more emulsifier was used.

Table 2 Diameter and polydispersion index of CPS prepared by using different amounts of emulsifiers

\begin{tabular}{ccc}
\hline Amount of emulsifiers, \% & Average diameter, $\mathrm{nm}$ & PDI \\
\hline 0.42 & 177.2 & 0.002 \\
0.59 & 142.9 & 0.021 \\
0.78 & 111.6 & 0.075 \\
\hline
\end{tabular}

Notes: Other preparation conditions: $10 \mathrm{wt} \%$ styrene, $0.05 \mathrm{wt} \% \mathrm{NaHCO}_{3}$, $0.05 \mathrm{wt} \% \mathrm{~K}_{2} \mathrm{~S}_{2} \mathrm{O}_{8}$ and $2.09 \mathrm{wt} \%$ DVB based on styrene

The glass transition temperature $(T \mathrm{~g})$ of the PS particles was measured with DSC. As shown in Fig. 4, the Tg of the PS particles was between $110{ }^{\circ} \mathrm{C}$ and $130{ }^{\circ} \mathrm{C}$, and the more crosslinker DVB that was used, the higher the $T \mathrm{~g}$ of the prepared CPS particles. However, because crosslinking only occurred on the shell of the particles, $\mathrm{Tg}$ did not change too much with the addition of crosslinker DVB.

To test their resistance to dissolution in organic solvents, the prepared CPS particles, and the PS particles, were put into tetrahydrofuran or toluene with a weight ratio of solvent to PS particles 10:1. The non-crosslinked PS particles completely dissolved in both solvents in five minutes, while the CPS particles did not dissolve at all, indicating that the CPS particles had much stronger solvent resistance than the noncrosslinked PS particles. $T \mathrm{~g}$ and the solubility tests indicated that the crosslinking process was successful.

The decomposition temperature of the PS particles was measured also by DSC and TG on the same thermal analyzer and the decomposition temperatures of different samples are shown in Table 3. Although the $T \mathrm{~g}$ of the PS particles varied with the degree of crosslinking, the decomposition temperature was almost invariable. The $T \mathrm{~g}$ of polymers not only depended on their chemical nature, but also on their molecular size, while the decomposition temperature depended mainly on their chemical nature.

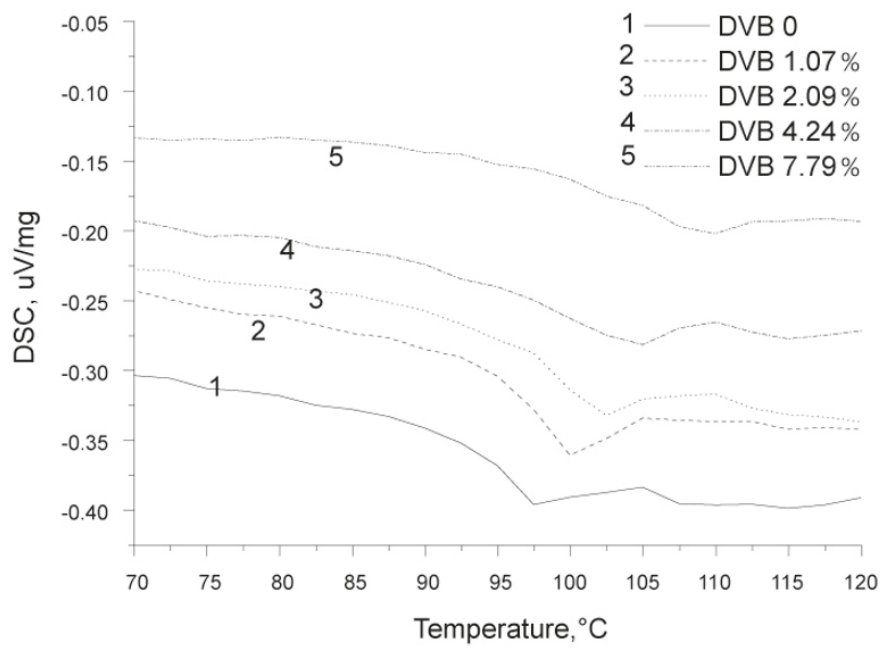

Fig. 4 DSC curve of CPS prepared with different amounts of crosslinker

Table 3 Effect of crosslinker on the decomposition temperature of PS particles

\begin{tabular}{ccccccc}
\hline Amount of DVB, wt $\%$ & 0 & 1.07 & $2.09^{*}$ & 4.24 & 7.79 & 31.8 \\
\hline Decomposition temperature, ${ }^{\circ} \mathrm{C}$ & 424.1 & 420.5 & 430.6 & 418.4 & 429.1 & 429.3 \\
\hline
\end{tabular}

Notes: *-DVB was added to the reaction system at the initiation, so the whole PS particles were uniformly crosslinked 


\section{Conclusions}

1) Crosslinked monodisperse PS particles were prepared by the emulsion method, which was previously used for the preparation of the non-crosslinked monodispere PS particles, by adding DVB crosslinker into the reaction system after PS particles grew to $\sim 80 \%$ of their final size.

2) When the crosslinker DVB was less than $6.17 \mathrm{wt} \%$ based on styrene, the prepared CPS particles were spherical and uniform, and the final size of the CPS particles was not affected by the DVB, and the particle size was the same as that of PS particles prepared with the normal emulsion method. If the crosslinker DVB was more than $7.79 \mathrm{wt} \%$, the PS particles aggregated during the synthesis and the formed particles were neither spherical nor uniform.

3) The glass transition temperature $(T g)$ of the prepared CPS particles was higher than that of the un-crosslinked PS particles and the more crosslinker that was used, the higher the $T \mathrm{~g}$, while the decomposition temperature of the PS particles remained unchanged.

4) The prepared CPS particles had much stronger solvent resistance than the non-crosslinked PS particles.

\section{Acknowledgements}

This research work was supported by National Basic Research Program of China (Grant No: 2006CB932601), and the Scientific Research Foundation for Returned Overseas Chinese Scholar, Ministry of Education.

\section{References}

Chen J W, Xu Z H and Jiang J L. Polymer Agents and Their Application.
Wuhan: Wuhan University Press. 1990. Chap. 4, 14-17 (in Chinese) Choi J, Kwak S Y, Kang S, et al. Synthesis of highly crosslinked monodisperse polymer particle :Effect of reaction parameter on the size and size distribution. Journal of Polymer Science Part A-Polymer Chemistry. 2002. 40(23): 4368-4377

Huang J X, Xu H J, Sun R, et al. Dispersion copolymerization of styrene/divinylbenzene in the mixture of ethanol and ethlene glycol monomethyl ether. Acta Polymerica Sinica. 2003. 4: 535-539 (in Chinese)

Jun J B, Hong J K, Park J G and Suh K D. Preparation of monodisperse crosslinked organic-inorganic hybrid copolymer particles by dispersion polymerization. Macromolecular Chemistry and Physics. 2003. 204(18): 2281-2289

Shim S E, Yang S H, Choi H H and Choe S. Fully crosslinked poly(styrene-co-divinylbenzene) microspheres by precipitation polymerization and their superior thermal properties. Journal of Polymer Science Part A: Polymer Chemistry. 2004. 42(4): 835-845

Xu M C, Shi Z Q and He B L. Synthesis Structures and Properties of Hypercrosslinked Polystyrene Adsorbents. Ion Exchange and Adsorption. 1994. 10(6): 555-562 (in Chinese)

You Y C, Qi D F, Zhang B L, et al. Studies on synthesis of monodispersed crosslinked polystyrene microspheres by seed polymerization. Polymer Materials Science and Engineering. 1994. 6: 13-17 (in Chinese)

Zhang H T, Huang J X, Jiang B B, et. al. Studies on kinetics of copolymerization and particle size for preparing monodispersed crosslinked polystere microspheres. Chinese Journal of Applied Chemistry. 2001.18(9): 726-730 (in Chinese)

Zhang H T, Jiang B B and Huang J X. Crosslinked polystyrene microsphere preparing by nonaqueous dispersion polymerizationEffect of monomer content on polymerization rate and particle size. Polymer Materials Science and Engineering. 2002.18(4): 57-61 (in Chinese)

(Edited by Zhu Xiuqin) 